\title{
Acoustic Characteristics of a Side Branch Silencer with the Finite Impedance at the End*
}

\author{
Masanori TSUJI** and Kunihiko ISHIHARA** \\ **Institute of Technology and Science, The University of Tokushima \\ 2-1 Minami-Jyosanjima-Cho, Tokushima-City, Tokushima-Pref. 770-8506, Japan \\ E-mail : ishihara@me.tokushima-u.ac.jp
}

\begin{abstract}
A side branch silencer which is one of the reactive-type silencers is effective for the reduction of periodical sound. It is often used to reduce noise such as those due to pulsation pressure. This is especially effective for noise generating a single frequency. However, an effective frequency range is comparatively narrow obliging the silencer to be enlarged as a countermeasure of low frequency sound. This may cause problems concerning installation space. In order to develop a side branch silencer which is effective at low frequency ranges, a side branch with the finite impedance at the end was made and examined. As a result, it was clarified that this side branch was useful for low frequency noise.
\end{abstract}

Key words: Sound and Acoustic, Noise Control, Modal Analysis, FEM, Wave, Side Branch Silencer, Acoustic Impedance

\section{Introduction}

A reactive silencer such as a side branch is effective in reducing periodic sound. Therefore, this silencer is especially effective for noise with a single frequency ${ }^{(1)} \sim(4)$. Moreover, because the structure is simple, the introduction of the silencer is comparatively easy. However, the effective frequency range is comparatively narrow. In addition, the silencer must be large enough to be a countermeasure against low frequency noise. This might cause problems concerning installation space when used.

Though there are some examples to reduce low frequency noise by applying a very long side branch in past research ${ }^{(5)}$. But, the size of the silencer becomes too large. Therefore, this strongly encourages the miniaturization of the silencer.

To solve the problem that this silencer has reduction effect in only the narrowband, Ojima et al. developed a type of side branch with a changeable resonance mode ${ }^{(6)}$. However, this type of silencer controls resonant frequency of a higher-order mode. It is not an effective counter measure for low frequency noise. In addition, one author of this paper clarified the reduction mechanism of the side branch silencer by measuring pressure fluctuations of the oil-hydraulic pump, and realized a drastic effect ${ }^{(7)}$. In this process, the reduction effect could not be obtained when using a rubber hose for the side branch. Consequently, we thought we would be able to change the frequency at which the noise would become smaller by softening the end of the side branch.

The objective of this study is to clarify that a shorter side branch could have a larger reduction effect for low frequency noise by also softening the end of the side branch. Therefore, an experimental setup was made and the experiment is conducted. Moreover, to use the silencer, it would be necessary to predict the noise reduction during the designing stage. Therefore, the reduction effect was predicted using the finite element method analysis. Furthermore, the sound pressure distribution and phase lag that had actually occurred in the silencer were examined under various conditions.

*Received 7 Apr., 2010 (No. T2-09-0672) Japanese Original : Trans. Jpn. Soc. Mech. Eng., Vol.76, No.762, C (2010), pp.282-289 (Received 17 July, 2009) [DOI: 10.1299/jee.5.456]

Copyright $\odot 2010$ by JSME 


\section{Experiment}

\subsection{Experimental setup}

The impedance was given by putting a rubber board on the end of the side branch in this experiment (Fig. 1). The impedance could be changed by altering the tension of the rubber board. The experiment was setup in a similar fashion. The sine wave is output from the speaker. The sound pressure level is measured at the back side of the main tube (Position of sound level meter in Fig. 1). The effect (Insertion loss: IL) of the silencer was obtained by measuring the sound pressure level with and without the silencer.

Fig. 2 shows a close up picture of the end of the side branch. The tension of the rubber board installed in a fixed plate is adjusted with a bolt. The vibration of the rubber board is measured with the laser displacement meter which can be traversed, and the sound pressure in the vicinity of the vibration plane is measured with the microphone set up inside the side branch. The impedance at the end is obtained through these data.

Moreover, the acoustic material is installed in the exit part of the main tube. As a result, the reflection is suppressed (Fig. 3 and appendix). The purpose of using the acoustic material is to eliminate the virtual reduction effect due to the standing wave.

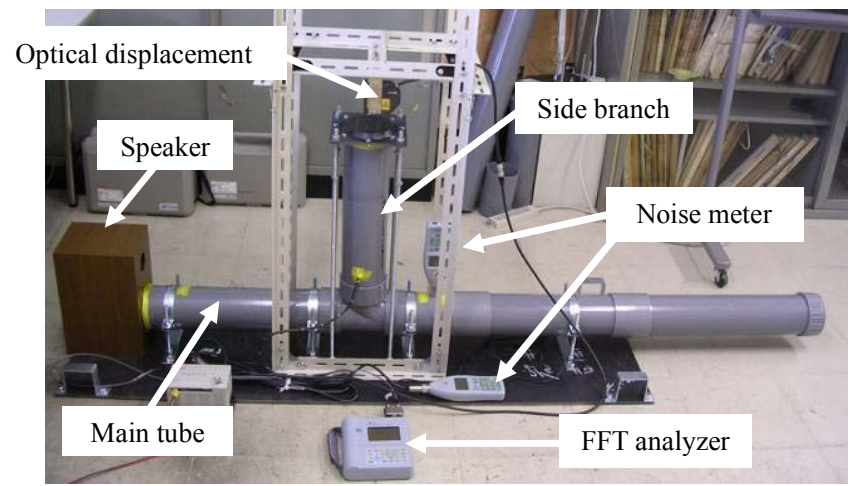

Fig. 1 Experimental setup (Integral)

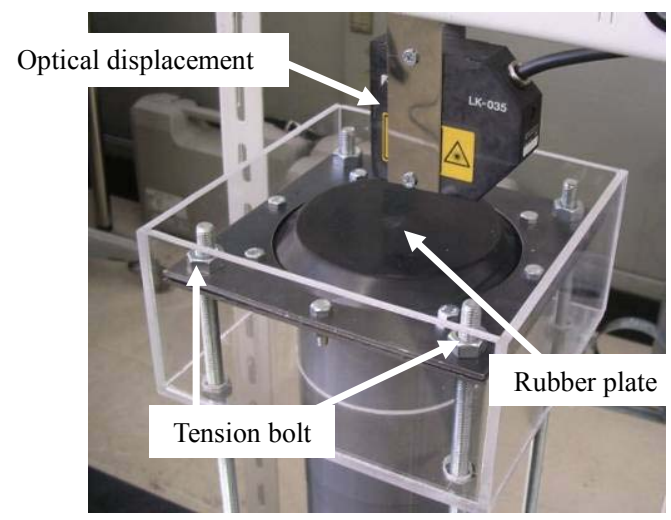

Fig. 2 Experimental setup (End of Side Branch)

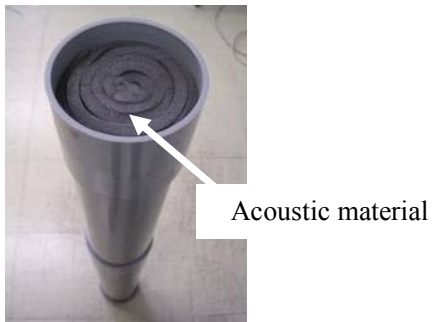

Fig. 3 Experimental setup (Acoustic material) 


\subsection{Experimental result}

The natural frequency at the end of the side branch (the circular membrane) can be changed from $70 \mathrm{~Hz}$ to $200 \mathrm{~Hz}$ at $10 \mathrm{~Hz}$ intervals by adjusting the tension of the rubber board. Furthermore, the insertion loss (IL) and the impedance at the end were measured.

IL is defined by $\mathrm{SPL}_{1}-\mathrm{SPL}_{2}$, in which the following can be said: $\mathrm{SPL}_{1}$ : Sound pressure level when the side branch does not exist. $\mathrm{SPL}_{2}$ : Sound pressure level when the side branch exists.

\subsubsection{Change in insertion loss upon alteration of natural frequency at the end}

Fig. 4 shows the insertion loss (IL) when the natural frequency at the end is $70 \mathrm{~Hz}$, $130 \mathrm{~Hz}, 200 \mathrm{~Hz}$ and about infinity (rigid end). When the end of the side branch is rigid, the peak frequency of IL is $165 \mathrm{~Hz}$. It is understood that the peak frequency shifts to the lower frequency side as the natural frequency of the end of the side branch is lowered. Moreover, the peak of a rigid end divided into different frequencies when the impedance was attached to the end. It also was understood that reduction effects of divided peaks were larger than the original one.

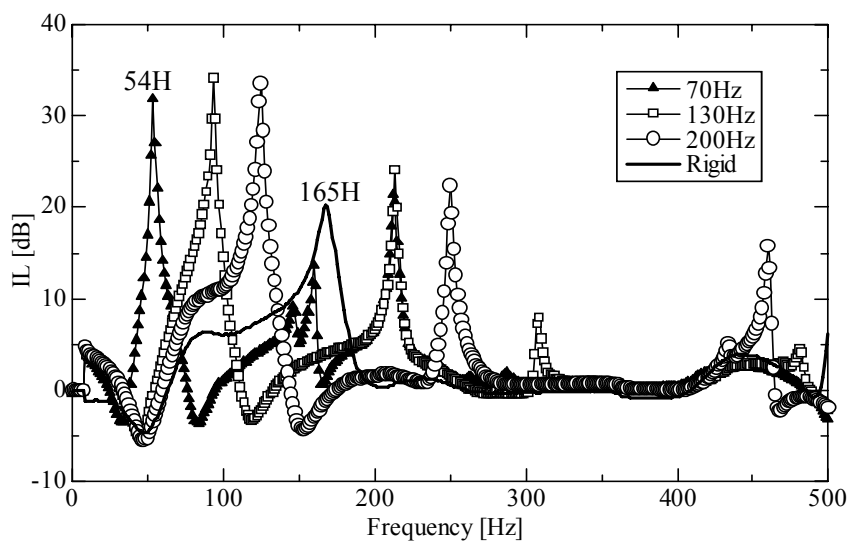

Fig. 4 Frequency response of Insertion Loss, IL

\subsubsection{Natural frequency at the end and peak frequency of IL}

The natural frequency at the end differed from the peak frequency of IL in the experiment as shown in Fig. 4. For example, when the natural frequency at the end was adjusted to $70 \mathrm{~Hz}$, the peak of IL appeared at $54 \mathrm{~Hz}$. The effect appeared at the lower frequency than the natural frequency at the end. The ratio of the peak frequency of IL on the lowest frequency side and the natural frequency at the end is shown in Fig. 5. The natural frequency at the end is shown in the horizontal axis and the ratio is shown in the vertical axis. The ratio of the natural frequency and the peak frequency is about 0.76 in the lowest natural frequency $(70 \mathrm{~Hz})$. On the other hand, the ratio is about 0.63 in the highest frequency $(200 \mathrm{~Hz})$ and the difference between the natural frequency and the peak frequency is increasing. Moreover, the ratio of the natural frequency and the peak frequency has become smaller linearly with the natural frequency at the end becoming larger.

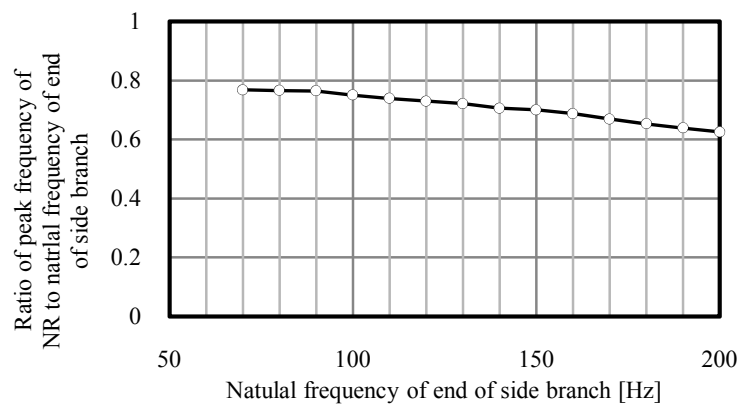

Fig. 5 Ratio of natural frequency of 01 mode and peak frequency of NR 


\subsubsection{Change in mobility at the end when the natural frequency altered}

The mobility at the membrane center when the natural frequency at the end is changed is shown in Fig. 6. The mobility is a physical value defined by velocity/sound pressure (Vibration velocity to unit force, where pressure is used instead of unit force), and it is the inverse of the impedance. Each natural frequency at the end corresponds to the peak of the mobility. Moreover, the natural frequencies of the second mode and the third mode can also be confirmed. It is thought that these are the natural frequencies of the mode with the circular node obtained from the vibration theory of a circular membrane. This experimental result will be compared in detail with the analytical one below in the post-paragraph.

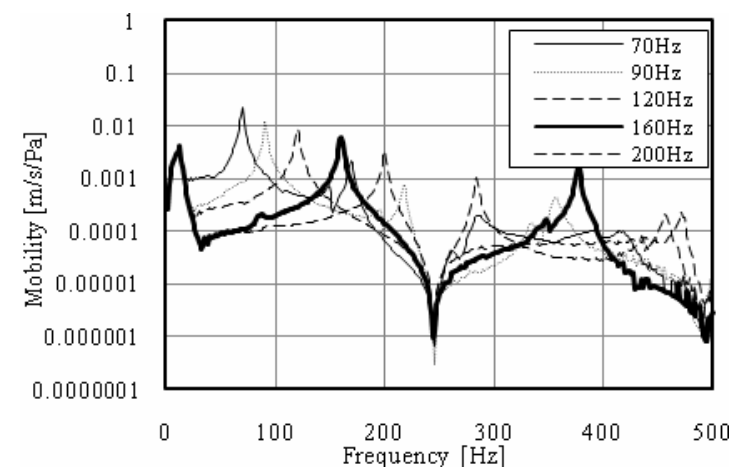

Fig. 6 Frequency response of mobility

\section{Analysis}

\subsection{Analysis method}

In order to examine whether the experimental results can be obtained by the analysis or not, the effect of the silencer was calculated by the two-dimension finite element method. Fig. 7 shows an analytical model.

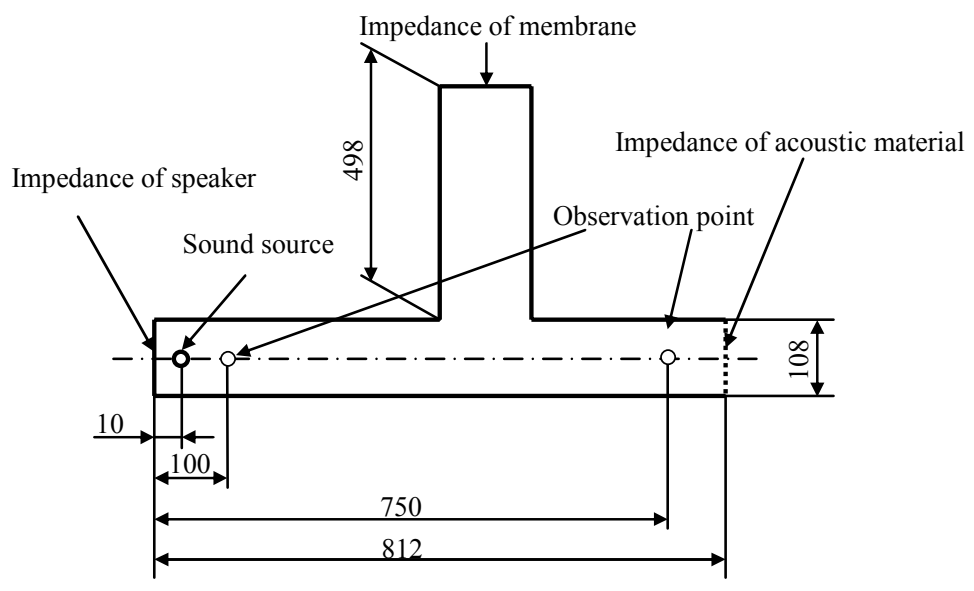

Fig. 7 Analytical Model

To express the speaker that was the sound source in the analysis, the impedance which was obtained by measuring the vibration on the speaker cone was given to the left end of the main tube. The point of the sound source was defined on the inside, $10 \mathrm{~mm}$ from the end. Similarly, the impedance of the acoustic material was given to the right end of the main tube. The vibration membrane impedance was given at the end of the side branch. Moreover, to use noise reduction (NR) as a reduction effect of the silencer in the analysis, 
the difference of the sound pressure level at two observation points as shown in Fig. 7 were measured.

\subsection{Analytical result}

In this section, the analytical and experimental results are compared.

\subsubsection{Theoretical mode shape}

When the impedance of a circular membrane is given in this analysis, the mode shape is taken into account (Impedance that varies from place to place is given). By doing so, an analytical result that was thought of to be near the actual phenomenon was obtained. First of all, the theoretical mode shape of the circular membrane was obtained by using the Bessel function. Following, the frequency response was obtained by superposing these mode shapes and using the modal analysis. Finally, the displacement and impedance at each position could be obtained, and they were compared with the experimental result.

For the first step, the displacement and compliance were obtained theoretically. The Bessel function is one of the solutions of the differential equation of the Bessel as shown by expression (1). $\alpha$ is an arbitrary real number (called the order).

$$
x^{2} \frac{d^{2} y}{d x^{2}}+x \frac{d y}{d x}+\left(x^{2}-\alpha^{2}\right) y=0
$$

When $\alpha$ is an integer or nonnegative in the differential equation of the Bessel, the singular solution in the $\mathrm{x}=0$ around becomes ${ }^{(8)}$.

$$
J_{\alpha}(x)=\sum_{m=0}^{\infty} \frac{(-1)^{m}}{m ! \Gamma(m+\alpha+1)}\left(\frac{x}{2}\right)^{2 m+\alpha}
$$

This is the first kind of Bessel function $J_{\alpha}(x)$. It is well known that this Bessel function shows the vibration of a circular membrane ${ }^{(9)}$. Especially, when the order of the Bessel function is assumed to be 0 , and the circle boundary condition of displacement is given 0 on the circumferences, the mode shape with a circular node is obtained. Moreover, when the smallest zero point (Intersection of Bessel function and $\mathrm{x}$ axis) of the 0th Bessel function is assumed to be a natural frequency of $(0,1)$ mode, the natural frequencies subsequent the $(0$, 2) mode are obtained by the ratio of the zero points of the Bessel function and the smallest zero point.

The mode is superposed by using each mode shape and the natural frequency obtained. Thus, the excitation coefficient $\beta_{i}$ is obtained by expression (3). Here $\boldsymbol{u}_{i}$ is the ith mode vector, $\boldsymbol{F}$ is the force vector, and $\boldsymbol{M}$ is the mass matrix.

$$
\beta_{i}=\frac{\boldsymbol{u}_{i}{ }^{T} \boldsymbol{F}}{\boldsymbol{u}_{i}{ }^{T} \boldsymbol{M} \boldsymbol{u}_{i}}=\frac{\boldsymbol{u}_{i}{ }^{T} \boldsymbol{F}}{M_{i}{ }^{*}}
$$

(However, $\quad \boldsymbol{u}_{i}{ }^{T} \boldsymbol{M} \boldsymbol{u}_{i}=M_{i}^{*}$ )

Moreover, $\xi_{i}$ is obtained by expression (4). Where $\omega$ is an angular frequency, $\omega_{i}$ is a natural angular frequency of ith mode, and $\zeta_{i}$ is a damping ratio of the ith mode.

$$
\begin{aligned}
& \xi_{i}=\frac{\beta_{i}}{(j \omega)^{2}+(j \omega) 2 \zeta_{i} \omega_{i}+\omega_{i}{ }^{2}} \\
&= \frac{\boldsymbol{u}_{i}{ }^{T} \boldsymbol{F}}{K_{i}{ }^{*}\left(1-\lambda_{i}{ }^{2}+j 2 \zeta_{i} \lambda_{i}\right)} \\
&(\mathrm{i}=1,2, \ldots, \mathrm{n}) \\
&\left(\text { However, }, \lambda_{i} \equiv \frac{\omega}{\omega_{i}}\right)
\end{aligned}
$$


From these, amplitude $\boldsymbol{X}$ of the steady response can be expressed as follows:

$$
\begin{aligned}
\boldsymbol{X}=\sum_{i=1}^{n} \frac{\boldsymbol{u}_{i}{ }^{T} \boldsymbol{F}}{K_{i}{ }^{*}\left(1-\lambda_{i}{ }^{2}+j 2 \zeta_{i} \lambda_{i}\right)} & \boldsymbol{u}_{i}=\boldsymbol{U} \boldsymbol{\xi} \\
& \left(\text { However, } \quad \boldsymbol{U}=\left[\boldsymbol{u}_{1}, \boldsymbol{u}_{2}, \boldsymbol{u}_{3}, \cdots, \boldsymbol{u}_{n}\right], \xi=\left(\xi_{1}, \xi_{2}, \xi_{3} \cdots \cdot, \xi_{n}\right)\right) .
\end{aligned}
$$

Fig. 8 shows the comparison between the experimental value and the theoretical value of compliance (displacement/pressure) in the case of the natural frequency at the end being $90 \mathrm{~Hz}$ (damping ratio is $\zeta_{1}=0.014, \zeta_{2}=0.025, \zeta_{3}=0.035$ and $\zeta_{4}=0.045$ ). The compliance at the center of a circular membrane is shown here as $(1,1)$ mode, $(1,2)$ mode, etc. with a straight line node in the direction of the diameter (nodal diameter mode) which was not seen in the experiment. This is due to expression (3) in which $\boldsymbol{u}_{i}{ }^{T} \boldsymbol{F}$ becomes 0 when the sound gives the sound pressure of the same amplitude and same phase to the circular membrane.

Therefore, it has been understood that only the mode shapes with a circular node are permitted to be superposed in theoretical calculations. When the theoretical values obtained here are calculated by superposing from $(0,1)$ mode to $(0,4)$ mode, the frequency of the $(0,1)$ theoretical mode was matched to the experimental value, and the theoretical value calculated by the above-mentioned method was compared with the experimental value. The frequencies of $(0,2)$ mode and $(0,3)$ mode are almost the same as shown in Fig. 8, and it can be said that the tendency of the theoretical frequency response is corresponding to the experimental one.

Moreover, the peak has divided into two sections at about the $(0,2)$ mode and $(0,3)$ mode in the experiment, and it has been understood that the mean value of two peak frequencies in each mode is almost corresponding to the theoretical value. As a result, it was confirmed that the theoretical calculation result was valid.

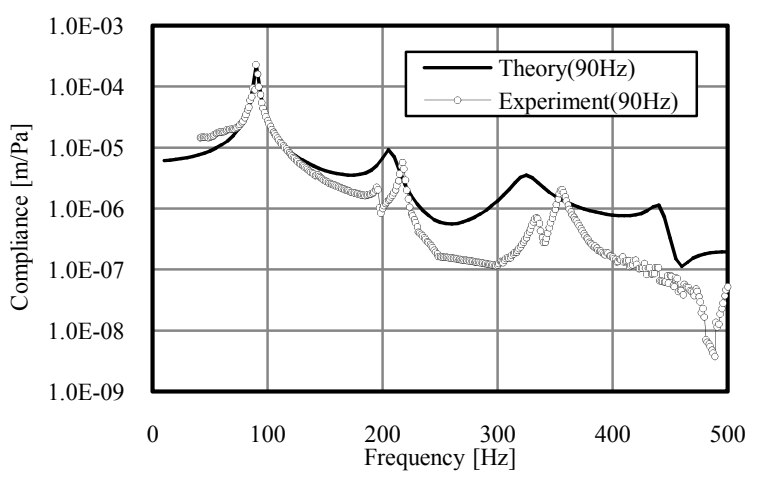

Fig. 8 Comparison of compliance

Fig. 9 shows the comparison between the theoretical result and the experimental one. The $(0,1)$ mode, $(0,2)$ mode and $(0,3)$ modes are shown. The theoretical mode shape is in very good agreement with the experimental one for the $(0,1)$ mode. On the other hand, two peaks were seen in the $(0,2)$ mode in the experiment. When both of two peaks were seen, the $(0,2)$ mode of $190 \mathrm{~Hz}$ was the shape to pull the theoretical mode shape radially. The $(0,2)$ mode of $211 \mathrm{~Hz}$ became the mode shape that rotated the one that had been seen in $190 \mathrm{~Hz}$ by $90^{\circ}$. As for this, the tension of the membrane is thought not to be the same in the circumference direction. Two peaks were able to be confirmed for the $(0,3)$ mode in the experiment, the same tendency as the $(0,2)$ mode was seen. However, two frequencies seen in $(0,2)$ mode and $(0,3)$ mode are extremely near the theoretical values respectively. Therefore, the mode shapes of both were corresponding well and it was thought that it was very possible to use the theoretical mode shape for the analysis. Then, the impedance was calculated by using the membrane vibration obtained by the theory, it was given to the end of the side branch, and the acoustic analysis was done. 

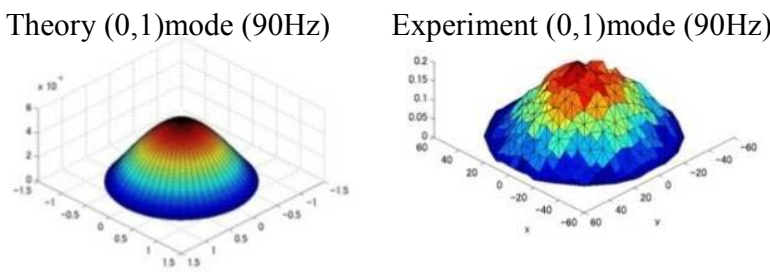

Experiment $(0,2)$ mode $1(190 \mathrm{~Hz})$
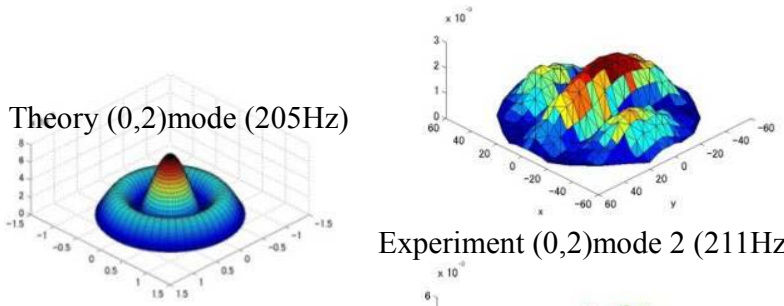

Experiment $(0,2)$ mode $2(211 \mathrm{~Hz})$

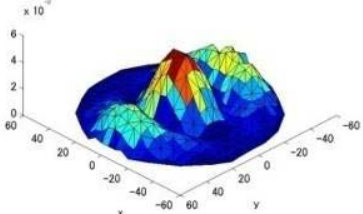

Experiment $(0,3)$ mode $1(335 \mathrm{~Hz})$
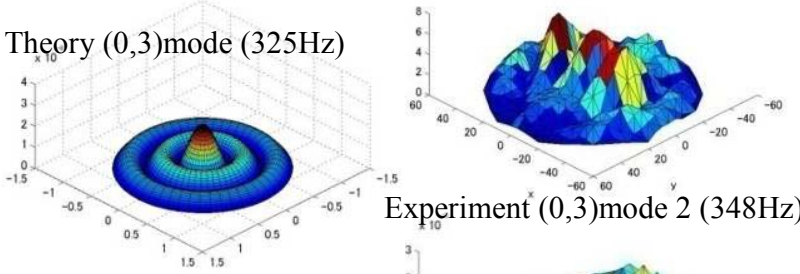

Experiment $(0,3)$ mode $2(348 \mathrm{~Hz})$

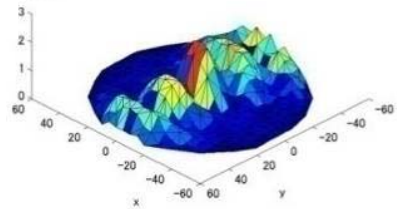

Fig. 9 Shape of mode

\subsubsection{Comparison of the analytical result with the experimental result}

The comparison of the analytical result with the experimental result of NR in the case of natural frequency at the end being $90 \mathrm{~Hz}$ is shown in Fig.10. An experimental and analytical value given impedance considering mode shape and analytical value when given impedance which doesn't consider the mode shapes are also shown here. As can be seen in Fig. 10, it is understood that the experimental value corresponds well to an analytical value surrounding the first peak frequency (about $70 \mathrm{~Hz}$ ). Though the peak frequency of the experimental value is $68 \mathrm{~Hz}$, it can be closely seen that the peak frequency considering mode shape was $65 \mathrm{~Hz}$ and not considering mode shape was $60 \mathrm{~Hz}$. That is to say, the analytical value in which the mode shape was considered is close to the experimental value. The peak does not appear in the analysis when the surrounding of the second peak frequency is seen. However, the analysis which considers the mode shape approaches the tendency to the experiment value.

Fig. 11 shows the comparison of the results when the natural frequency of the membrane is $170 \mathrm{~Hz}$. An analytical value that considers mode shape and the experiment value completely correspond around $115 \mathrm{~Hz}$ which is the first peak. Moreover, even if the mode shape is not considered, the gap lessens. Moreover, an analytical value and the experiment value correspond well for the second peak. The experimental value of $170 \mathrm{~Hz}$ overall corresponds more to an analytical value compared with the case when the natural frequency of the membrane is $90 \mathrm{~Hz}$ (Fig. 10). The experimental value corresponds very well to the theoretical value of compliance for the peak of the $(0,1)$ mode. When the natural frequency 
of the $(0,1)$ mode of the membrane is $90 \mathrm{~Hz}$, the range of the frequency where the theoretical value is corresponding to the experimental value is narrow. On the other hand, when the natural frequency of $(0,1)$ mode of the membrane is $170 \mathrm{~Hz}$, the range in which the theory and the experiment corresponds broadens because the range of the frequency of the $(0,1)$ mode is wide. The NR is also thought to be in agreement comparatively well.

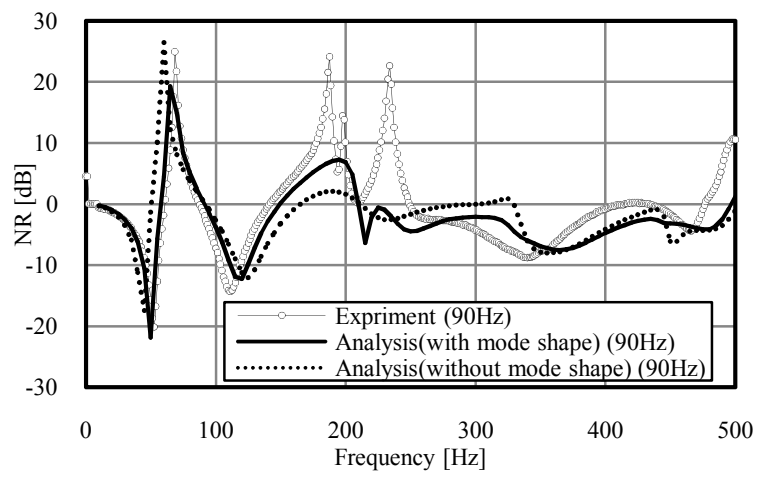

Fig. 10 Comparison of Experimental result and Analytical result

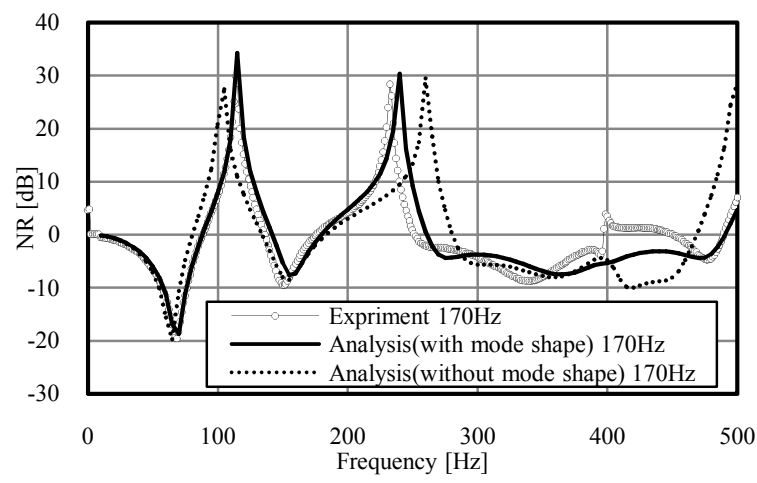

Fig. 11 Comparison of Experimental result and Analytical result

\subsubsection{Impedance and sound pressure distribution}

The experimental value matches well with the analytical result. It is thought that the analysis illustrates the actual phenomenon. The influence of the impedance on the sound field will be examined by using a theoretical impedance and sound pressure distribution in a model obtained by the analysis.

It is known that the impedance is generally expressed by the complex number; the real part acts as the attenuation element, and the imaginary part acts as a stiffness element. The real and imaginary parts are given at the same time for the impedance of a circular membrane in this study. It becomes important to know how these impedance parts act with each other. We tried to analyze the simple acoustic tube as shown in Fig. 12 where the impedance is given at the end of the tube. Each influence of a real part and an imaginary part of the impedance is considered by focusing on resonant frequency.

The two-dimensional finite element method is used for this analysis. The length of the tube is $1.0 \mathrm{~m}$; a left end-part is assumed to be a closed end, and the impedance is given to the other end. Because the tube length is $1.0 \mathrm{~m}$, the resonant frequency of this tube becomes $170 \mathrm{~Hz}$ for the boundary conditions of both ends are closed. It becomes $85 \mathrm{~Hz}$ when one end is closed and the other end is open.

Fig. 13 is the analytical result showing the relation between the impedance ratio given to the end and the resonant frequency. The real part $Z_{R}$ of the impedance ratio is shown in a left horizontal axis. The imaginary part $\mathrm{Z}_{\mathrm{i}}$ of the impedance ratio is shown in the right horizontal axis. The resonant frequency $f_{n}$ is shown in the vertical axis. When the real part 
and the imaginary part are changed, it is understood that the resonant frequency changed within the range of $85 \mathrm{~Hz} \sim 170 \mathrm{~Hz}$. When a real part of the impedance ratio is larger than 1.0, the resonant frequency is a constant value $(170 \mathrm{~Hz})$ regardless of the value of the imaginary part. When a real part is 1.0 or less, the resonant frequency continuously changes from $85 \mathrm{~Hz}$ to $170 \mathrm{~Hz}$ whenever the imaginary part is changed.

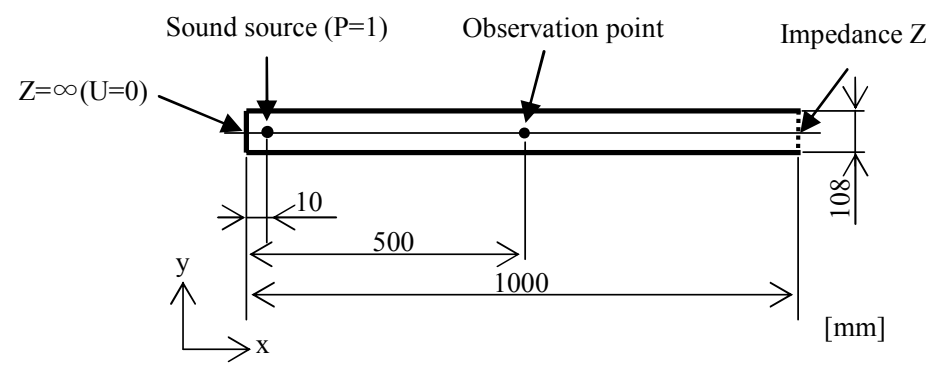

Fig. 12 Analytical Model of acoustic tube with impedance

Fig. 14 shows a real and an imaginary part of the impedance ratio obtained by the theory when the natural frequency of the membrane is $200 \mathrm{~Hz}$ and the NR is obtained by analysis. The solid line shows NR, a black circle $(\bullet)$ shows a positive value of real part of an impedance ratio, a black triangle $(\boldsymbol{\Delta})$ shows a negative value of the real part of an impedance ratio, a white circle $(\circ)$ shows a positive value of imaginary part of impedance ratio, and a white triangle $(\triangle)$ shows a negative value of an imaginary part of an impedance ratio.

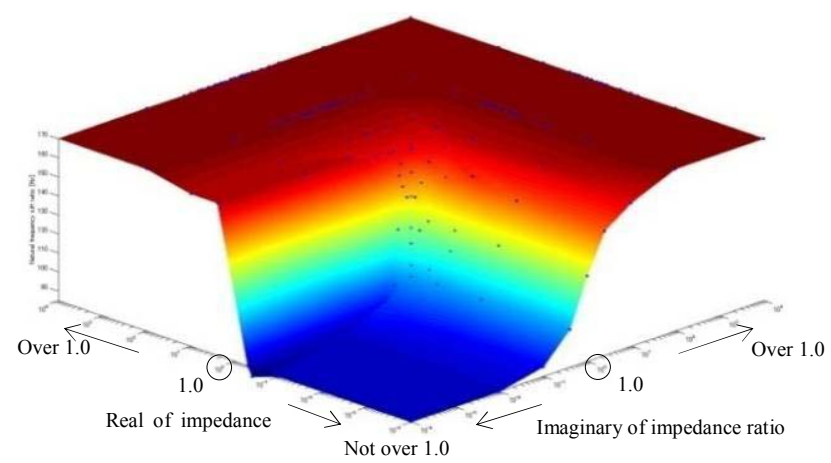

Fig. 13 Impedance ratio and natural frequency

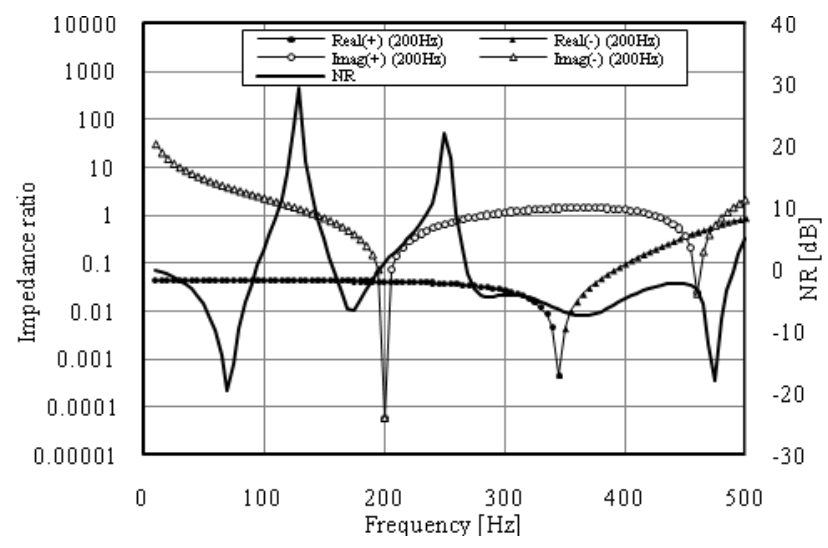

Fig. 14 Noise reduction and Impedance ratio

As can be seen in Fig. 14, a real part of the impedance ratio is 1.0 or less within the range of all frequencies. Therefore, it is understood that the internal acoustic field (especially resonant frequency) changes when the value of the imaginary part of the impedance ratio changes. Moreover, the sign is reversed at each reverse point of the peak impedance, and it 
can be expected that the state of the sound field is especially different before and behind the peak frequency of the imaginary part of impedance ratio.

Fig. 15 shows the sound pressure distribution in the first peak frequency $(130 \mathrm{~Hz})$ obtained by the analysis. The distance from the side branch bifurcation part is shown in a horizontal axis, and the sound pressure is shown in the vertical axis in Fig. 16. The sound pressure distribution in the direction of the side branch from the side branch bifurcation part was compared with the sound pressure distribution in the direction of the sound source is shown. Fig. 15 shows the sound pressure distribution by the gray scale. The sound pressure is high when the color is dark, and the sound pressure is near $0 \mathrm{~Pa}$ when the color is light. Moreover, the sound pressure distribution along the side branch is shown by the solid line. The belly of the virtual acoustic pressure is shown in the dotted line.

The acoustic pressure is almost $0 \mathrm{~Pa}$ on the exit side of the main tube, and it is understood that the effect of the side branch has been brought sufficiently. However, it doesn't become a sound pressure distribution that the end of the side branch becomes the belly of the sound pressure in the frequency where the reduction effect maximizes which is a feature of the side branch silencer. When the acoustic pressure distribution of the branch part is seen in Fig. 14, the sound pressure is at a maximum at the end. However, it is not the complete belly of the sound pressure at the end. It is the side branch which is virtually expanded as the reflection, and is delayed by the action of the impedance at the end of the side branch.

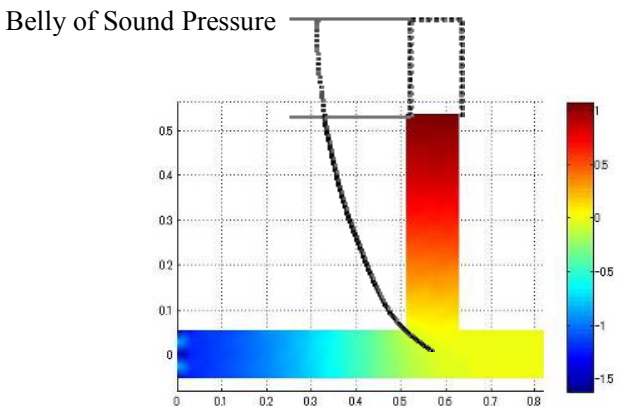

Fig. 15 Sound pressure distribution on 1 st peak frequency $(130 \mathrm{~Hz})$

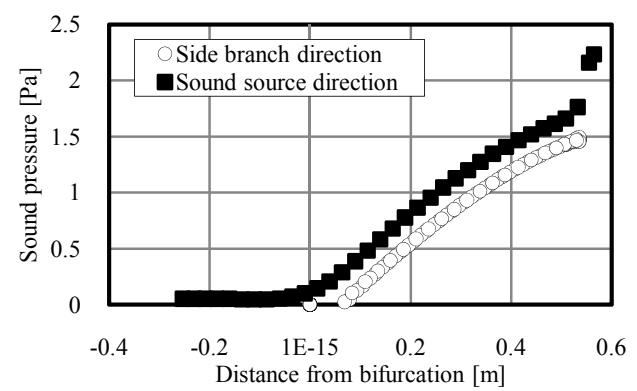

Fig. 16 Sound pressure distribution from bifurcation $(130 \mathrm{~Hz})$

Fig. 17 and Fig. 18 show the sound pressure distributions of $250 \mathrm{~Hz}$ that is the second peak frequency. The sound pressure distribution of Fig. 17 is different from that of Fig. 15, and the belly of the sound pressure is seen on the way in the side branch. The side branch is virtually shortened as the reflection progresses through action of the impedance at the end of the side branch.

When you compare the imaginary part of the impedance ratio $(\circ, \triangle$ in Fig. 14) shown in Fig. 14 and these results, it is clarified that it is in the state that the branch virtually expands when the imaginary part impedance is positive, and it is in the state that the branch virtually shortened when the imaginary part impedance is negative. 


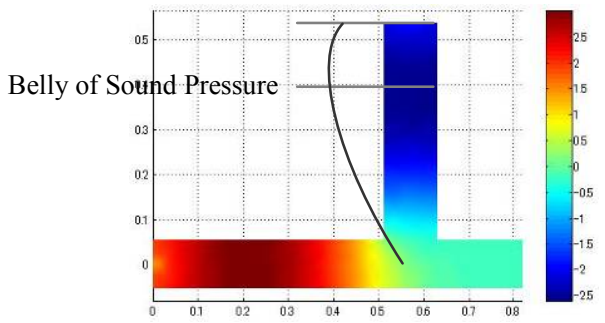

Fig. 17 Sound pressure distribution on 2nd peak frequency $(250 \mathrm{~Hz})$

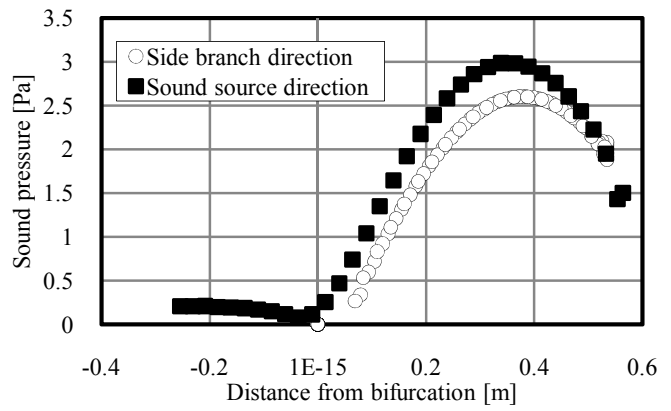

Fig. 18 Sound pressure distribution from bifurcation $(130 \mathrm{~Hz})$

\subsubsection{Difference between natural frequency of the membrane and peak frequency of} NR

Let's consider the cause of the difference between the natural frequency of the membrane and the peak frequency of NR. Fig. 19 shows the analytical result when the side branch length is changed and the natural frequency of the membrane is constant. This figure shows the analytical result in the case of the side branch length being $0.8 \mathrm{~m}, 0.535 \mathrm{~m}$, and $0.054 \mathrm{~m}$ (state without side branch) when the natural frequency of the end is $70 \mathrm{~Hz}$.

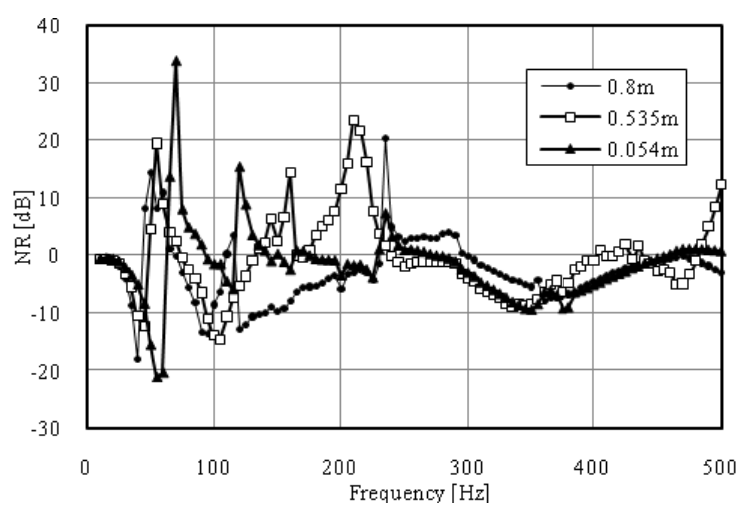

Fig. 19 Noise reduction of silencer on several side branch length

When the side branch length is $0.054 \mathrm{~m}$ (state without the side branch), the first peak frequency is $70 \mathrm{~Hz}$. It also corresponds to the natural frequency of end $(70 \mathrm{~Hz})$. Therefore, it is understood that the reduction effect appears by only the membrane even if there is no side branch. Moreover, when the side branch length is $0.535 \mathrm{~m}$, the peak $(70 \mathrm{~Hz})$ falls to $55 \mathrm{~Hz}$ after a while. It is because reflection is delayed due to the side branch expanding, and the peak frequency falling to $50 \mathrm{~Hz}$ when the side branch is extended to $0.8 \mathrm{~m}$. The reason why the peak frequency of the effect does not correspond to the natural frequency of the end is not only due to the vibration of the membrane but also the length of the side branch which is effective in the phase delay of the reflected sound. As a result, it is thought that the combination of the side branch length and the vibration membrane is effective for the reduction of low frequency noise. However, when we want to reduce the low frequency noise, the change in branch length is not effective. That is to say, wavelengths become long, 
and since a shift to an effective frequency does not appear easily only from a few changes to the length of the side branch. In low frequencies, lowering the natural frequency of the vibration membrane becomes more effective than extending the side branch length.

\section{Conclusion}

The acoustic characteristic of finite impedance given at the end of the side branch was investigated analytically and experimentally in order to improve the applicability of a side branch type silencer. The following findings were obtained.

(1) The peak of NR when the end is rigid divides into peaks when impedance is given to the end of the side branch. Furthermore, the effect extends to a comparatively wide frequency. Moreover, it could also be confirmed that the reduction effect becomes larger.

(2) When you want NR given impedance at the end of the side branch as the analysis, the imaginary part of the impedance is essential, and it is effective to take into account the mode shape of the vibration membrane.

(3) When the impedance is given at the end of a side branch, reduction effects appear at a frequency lower than the peak frequency in the case of a rigid end. This is a phenomenon of the side branch virtually becoming long. The effect seen in a high frequency is a phenomenon of the side branch virtually shortening.

(4) The phenomenon which the peak frequency of the impedance differs from the peak frequency of NR. This was able to be confirmed in both the experiment and the analysis. The difference is decided by the mutual influence of the natural frequency at the end and the side branch length.

Future work warrants application to a real machine. The strength at the end with impedance and radiation of sound seem to become problems when applying it to a real machine. The end will become a vibration system composed by material with large transmission loss such as iron plates in the future.

\section{Appendix: Influence on acoustical property of acoustic material}

The influence of the acoustic material used in the present experiment on the acoustic characteristic of tubes is discussed. Fig. A1 shows the acoustic characteristics when the acoustic material was given at the end part of a simple tube of $0.91 \mathrm{~m}$ and not given. When the acoustic material is not given, the resonances of the first mode and the second mode are clearly seen. On the other hand, the value has become smaller (about 20dB) in the first mode though the resonant frequency changes when the acoustic material is applied to the end part. From this result, the main tube can be regarded as the tube with the infinite length to apply the acoustic material at the end of the tube.

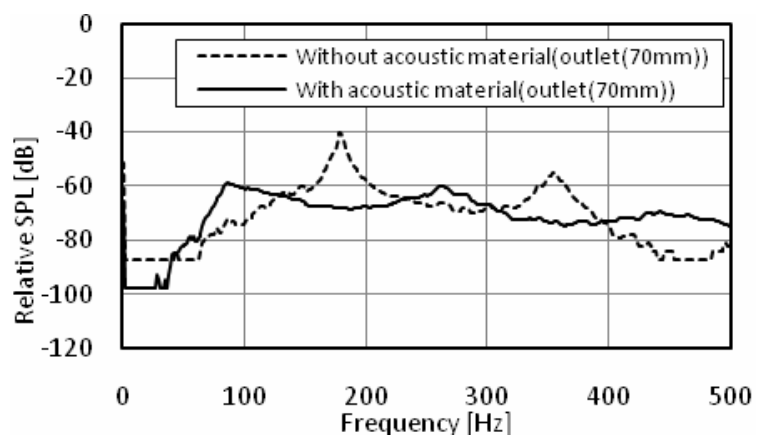

Fig. A1 Acoustic characteristic of simple pipe 


\section{References}

[1] Kazuhiro SHIRAKI, Anti-noise design and Simulation, (1987), Ohyogijyutsu Publication, p200.

[2] Masanori TSUJI and Kunihiko ISHIHARA, Study on Noise Reduction of Suction machine with Complicated Piping System by use of Side Branch, The 37th International Congress \& Exhibition on Noise Control Engineering, (2008), in CD ROM.

[3] Masanori TSUJI, Michiya OKAMURA and Kunihiko ISHIHARA, On Noise Reduction Effect of Side Branch in Complex Piping System (Comparison between Results of BEM and Experiment), The 46th term general meeting, Lecture collected papers of JSME, Chugoku and Shikoku branch division, (2008), pp.287-288.

[4] Michiya OKAMURA, Kunihiko ISHIHARA and Masanori TSUJI, Study on Noise Reduction of Suction machine with Complicated Piping System by use of Side Branch, JSME 2008 Symposium on Environmental Engineering; (2008), pp.135-138.

[5] Environmental Protection Bureau Environment Room of Atmospheric Life, Low Frequency Sound Prevention Measures Case Collection, (2002), http://www.env.go.jp/air/teishuha/jirei/.

[6] Eiichi KOJIMA, Yoshikazu KIMURA, Tatsushi TERASAWA and Seiishiro TAKESHITA, Development Research of Variable Resonance-Mode Type of Side-Branch Resonators, Transactions of the Japan Society of Mechanical Engineers, Series C, Vol. 64, No.621 (1998), pp.120-127.

[7] Kunihiko ISHIHARA, Yuichi MORIGUCHI and Kazuo NISHIKAWA, A Consideration of reduction Effect of Side Branch on Pressure Fluctuation, Transactions of the Japan Society of Mechanical Engineers, Series C, Vol. 70, No.690 (2004), pp.394-399.

[8] Tadao KUBO, Basic of Applied Mathematics, (1963), Kyoritsu Syuppan, p.159.

[9] Yasuaki TOMIHISA, Advanced Engineering Mathematics, (1960), Brain Book Publication, p.486

[10] Akio NAGAMATSU, Dynamics Handbook, (1993), Asakura Bookstore, pp.113-114. 